\title{
Multifocal primary salivary-type adenocarcinoma of the esophagus
}

An 83-year-old woman presented with a history of dysphagia and a $10-\mathrm{kg}$ weight loss over a 6-month period. On endoscopy, multiple glassy-looking polyps were seen in the upper and middle thirds of the esophagus (Figure 1a). No Barrett's metaplasia was observed in the distal esophagus. Biopsy specimens taken from the polyps revealed multifocal adenocarcinoma originating from the ducts of esophageal mucus glands. The lesions showed extensive mucus production and were located mainly in the submucosal layer, undermining non-neoplastic squamous epithelium (Figure $\mathbf{1} \mathbf{b}$ ). There were micropapillary in-situ formations as well as foci of overt, infiltrating, poorly differentiated cancer (Figure 1c). The patient refused further evaluation and therapy.

Ten months later she presented again with dysphagia. The tumor had progressed to cause almost complete luminal obstruction, with coalescing, exophytic, friable masses throughout the entire esophagus, with infiltration of the gastric cardia (Figure 1d). Computed tomography showed that there were multiple metastases to regional lymph nodes, the lungs, and bone. The patient's general condition worsened rapidly and she died 11 months after the primary diagnosis.

The vast majority of adenocarcinomas of the esophagus arise in the lower third, within a segment of Barrett's mucosa. Salivary-type adenocarcinomas originating from submucosal esophageal glands are extremely rare [1]. It has been argued, however, that the true incidence of these tumors is higher than has been reported because of missed diagnoses due to endoscopic tissue sampling [2]. Because of the rarity of the disease, the impact of treatment on survival is difficult to assess. Local ablative therapy and radiotherapy might offer palliation of dysphagia in patients with advanced disease [3].

Most esophageal salivary-type malignancies are adenoid-cystic and mucoepidermoid carcinomas [1]. Our case did not fit

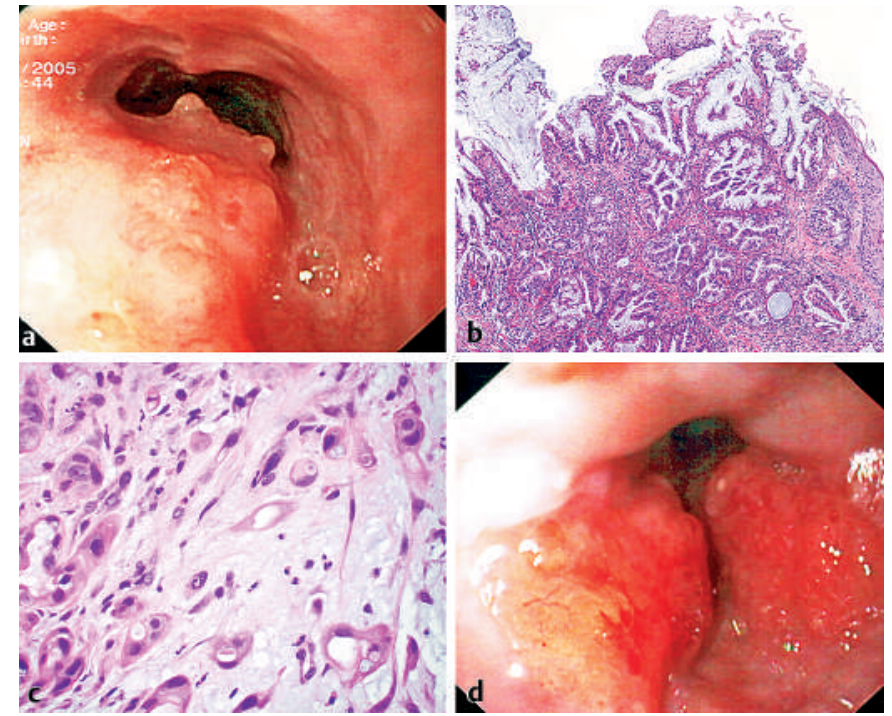

Figure 1 a Endoscopy showed a glassy polypoid tumor in the middle third of the esophagus with mucus drainage from the surface. $\mathbf{b}$ The corresponding biopsy specimen revealed a welldifferentiated adenocarcinoma with extensive mucus production. c Small tumor foci were shown to be poorly differentiated adenocarcinoma with single-cell invasion of the adjacent stroma. d Ten months later, an exophytic, friable esophageal tumor mass causing almost complete luminal obstruction was seen on endoscopy.

into either category and had to be classified as "adenocarcinoma of salivary duct origin, not otherwise specified (NOS)". The multifocal growth along the entire organ in this case is a tumor feature that has not been described before.

Endoscopy_UCTN_Code_CCL_1AB_2AC_3AB

\section{J. Pollheimer ${ }^{1}$, V. S. Pollheimer ${ }^{1}$,}

\section{A. J. Eherer ${ }^{2}$, C. Langner ${ }^{1}$}

${ }^{1}$ Institute of Pathology, Medical University of Graz, Graz, Austria

2 Department of Internal Medicine, Medical University of Graz, Graz, Austria.

\section{References}

\footnotetext{
${ }^{1}$ Werner M, Flejou JF, Hainaut P et al. Adenocarcinoma of the oesophagus. In: Hamilton SR, Aaltonen LA (eds). Pathology and genetics: tumours of the digestive system. Lyon: IARC Press, 2000: 20-26

2 Batoon SB, Banzuela M, Angeles HG et al. Primary mucoepidermoid carcinoma of the esophagus misclassified as adenocarcinoma on endoscopic biopsy. Am J Gastroenterol 2000; 95: $2998-2999$
}

${ }^{3}$ Karaoglanoglu N, Eroglu A, Turkyilmaz A et al. Oesophageal adenoid cystic carcinoma and its management. Int J Clin Pract 2005; 59: $1101-1103$

\section{Corresponding author}

\section{Langner, MD}

Institute of Pathology

Medical University of Graz

Auenbruggerplatz 25

A-8036 Graz

Austria

Fax: $\quad+43-316-384329$

Email: cord.langner@meduni-graz.at 